\title{
Rapid, risk-based levee design framework for greater risk reduction at lower cost than standards- based design
}

\author{
David Johnson ( $\square$ davidjohnson@purdue.edu ) \\ Purdue University https://orcid.org/0000-0002-2364-340X \\ Jingya Wang \\ Purdue University
}

Nathan Geldner

Purdue University

Andrew Zehr

Purdue University

\section{Article}

Keywords: Storm Surge, Interior Dynamics, Risk-informed Design Heights, Multi-objective Evolutionary Algorithms, Larose to Golden Meadow Hurricane Protection Project

Posted Date: February 23rd, 2021

DOl: https://doi.org/10.21203/rs.3.rs-252734/v1

License: (c) (i) This work is licensed under a Creative Commons Attribution 4.0 International License. Read Full License

Version of Record: A version of this preprint was published at Journal of Flood Risk Management on February 11th, 2022. See the published version at https://doi.org/10.1111/jfr3.12786. 


\title{
Rapid, risk-based levee design framework for greater risk reduction at lower cost than standards- based design
}

\begin{abstract}
Standards-based levee design aims to protect against events with specific probabilities, for example eliminating overtopping from a "1-in-100-year" storm surge. This allows levee segments to be analyzed independently but ignores interior dynamics and overall risk. We present and implement a framework for calculating optimal risk-informed design heights. Using this design paradigm and multi-objective evolutionary algorithms, we identify levee and floodwall design heights that minimize the total system cost and expected flood losses over 50 years. With our model, decision makers may feasibly evaluate hundreds or thousands of alternative designs. Comparing to the existing design elevations of the Larose to Golden Meadow Hurricane Protection Project in coastal Louisiana, over multiple climate change scenarios, we identify system configurations of similar cost that reduce the expected value of discounted residual risk of $26-73 \%$ ( $\$ 8-85$ million). We also achieve the same residual risk at $90-97 \%$ of the cost of the existing system (saving \$19-73 million).
\end{abstract}

\section{Introduction}

Coastal flood risk management efforts in the United States commonly adopt a standards-based approach ${ }^{1,2}$. Analysts estimate a standard design load, such as the 100-year storm surge (a peak surge elevation with a 1in-100 chance of occurring or being exceeded in a given year). Structural protection systems (e.g., levees, floodwalls) are then designed with crest heights that withstand the design load with some specified level of performance. For example, the U.S. Army Corps of Engineers (USACE) might set crest heights using a design criterion specifying that "the [maximum] overtopping rate should be less than $0.1 \mathrm{cfs} / \mathrm{ft}$ with 90 percent assurance"3. USACE guidance formally regards this kind of "level of protection" as a legacy term and requires risk assessment of any design alternatives under consideration ${ }^{4,5}$. However, in practice, 
communities requesting feasibility studies for structural protection often opt for a 100-year, standardsbased design as providing minimal protection to be considered "outside of the designated floodplain" and avoid requirements of the U.S. National Flood Insurance Program. This approach is tractable, interpretable, and intuitive, allowing analysts to treat each point along the system boundary independently. However, it does not provide any assurances about the remaining risk to assets the system is designed to protect. Further, reducing 100-year overtopping to a negligible rate all along the system boundary does not even guarantee that 100-year flood depths on the interior will be negligible, due to nonlinearities in overtopping and fragility dynamics and consideration of rainfall and pumping behavior ${ }^{6}$. Cost-effective flood risk mitigation therefore requires a risk-based analysis--one which is driven by the probability distribution of flood depths and consequences on the system interior, rather than hazard on the boundary ${ }^{7}$ This can be achieved by optimizing economics-based objective functions, such as (i) simultaneously minimizing the system cost and minimizing expected losses (i.e., Pareto frontier), (ii) simultaneously minimizing system cost and losses with various annual exceedance probabilities, or (iii) maximizing the system's net present value (i.e., risk reduction benefits minus project cost) ${ }^{8,9}$.

Existing models of surge- and wave-induced flooding are either sufficiently detailed for standards-based planning and design, or computationally efficient enough to optimize risk reduction, but not both. While risk-based policy analysis for the management of natural hazards risks and natural resources has become increasingly prevalent in recent years, it has been used primarily in relation to water quality ${ }^{10-15}$. Past studies have addressed the risk-based design of protection systems, as early as van Dantzig in $1956^{16}$. However, prior analyses make simplifying assumptions that limit their usefulness for real-world application, such as (i) ignoring dynamics such as wave overtopping, rainfall, interior pumping, or levee failures; (ii) modeling levees/dikes as rings with uniform crest heights; (iii) assuming a single, often catastrophic, value for stillwater elevations or damage if overtopping occurs; (iv) ignoring future economic growth or changes to the vulnerability of exposed assets; (v) assuming stationarity in climate conditions such as future storminess; and/or (vi) applying a bathtub model of sea level rise rather than explicitly 
modeling surge/waves as a function of storm parameters and sea level ${ }^{8,9,16-23}$. Due to the complexity and computational requirements of modeling storm surge and wave hydrodynamics and their interactions with engineered systems, risk-based approaches to levee design have heretofore used statistical representations of surge hazard rather than process-based physical simulations of tropical cyclones. Some research demonstrates sophisticated approaches to optimizing risk reduction strategies but limits their implementation to an abstract representation rather than a real context ${ }^{24}$. USACE regulations require risk analysis to estimate the benefit-cost ratio of proposed projects ${ }^{4}$, but models used for this purpose (e.g., HEC-FDA) make other simplifying assumptions ${ }^{25}$. Therefore, existing modeling frameworks do not permit optimization or an effective search of the space of possible interventions; analysts instead construct and compare design alternatives based on simple assumptions about how 100-year protection standards may change over time due to factors like sea level rise. In this analysis, we introduce a process-based model that overcomes these simplifications and limitations.

The model introduced in this paper, the Surge and Waves Model for Protection Systems (SWaMPS), maintains sufficient realism for practical application while achieving sufficient computational efficiency to enable risk-based design in the domain of coastal flooding. SWaMPS simulates the physical processes of surge/wave overtopping, rainfall, pumping, system fragility, and climate change to evaluate flood risk within a single-polder ring levee system. For any specified set of environmental conditions, it runs Monte Carlo simulations for 120 synthetic tropical cyclones, aggregating the results into an annual exceedance probability distribution using joint probability methods ${ }^{26-28}$. Critically, SWaMPS is four orders of magnitude faster than a similar model, the Coastal Louisiana Risk Assessment (CLARA) model used to support recommended investments in structural and nonstructural risk mitigation measures as part of the State of Louisiana's Coastal Master Plan ${ }^{26,29}$. Thousands of designs can be evaluated rapidly, enabling levee and floodwall design heights to be optimized against an [economic] objective function(s) in a computationally feasible time frame. 
In this analysis, we simultaneously minimize the cost of system upgrades and the present value (PV) of expected losses over a 50-year planning horizon. We utilize the Borg multi-objective evolutionary algorithm $^{30,31}$, the NSGA-II genetic algorithm ${ }^{32}$ and a greedy algorithm that selects incremental upgrades producing the greatest marginal cost effectiveness. While the model and optimization frameworks are designed to be portable to other poldered (i.e., enclosed) protection systems, the initial application presented in this paper is the Larose to Golden Meadow Hurricane Protection Project in coastal Louisiana, where we optimize the crest heights of the twelve levee reaches defined by the South Lafourche Levee District $^{33}$. For simplicity, we focus on exploring the impact of climate change factors on risk-based levee designs, but we note that SWaMPS also permits variation in population/economic growth, discount rates, the planning horizon, and changes to building codes affecting vulnerability (e.g., raising first-floor elevations).

\section{Results}

We first examined the performance of potential system designs in four modeled future states of the world; the first assumes stationary (i.e., constant) risk over time, and the other three represent the assumptions of the Low, Medium, and High environmental scenarios from Louisiana's 2017 Coastal Master Plan ${ }^{34}$. These scenarios vary in their assumptions about future sea level rise and changes to the average intensity and frequency of tropical cyclones impacting the Louisiana coast. They were originally informed by the best available science at the time $\mathrm{e}^{35-48}$, but we have also chosen them for their salience to policy makers, and to explore the sensitivity of optimal design heights to assumptions about nonstationary hazards; we do not make assertions about the likelihood of any one scenario coming to pass. The parameter values in Year 50 corresponding to each scenario are summarized in Table 1.

The left-hand pane of Figure 1 illustrates the Pareto frontier of nondominated strategies that minimize the system cost (e.g., planning and design, construction, and maintenance) and the discounted present value of expected damage to assets within the polder, both over a 50-year time horizon. For this analysis, we consistently apply a $3 \%$ annual discount rate to convert future cash flows to their present value. This value 
was chosen as approximately equal to the rates used in recent years by the US Army Corps of Engineers when evaluating flood protection projects ${ }^{49}$. The four shapes correspond to the frontiers of the four future scenarios. Light gray marks indicate the cost and performance of the existing system's design heights (located on the horizontal axis at $\$ 728$ million). The actual Larose to Golden Meadow project began construction in 1972 and underwent periodic construction over the next four decades. In order to make a consistent cost comparison, we show estimates from the SWaMPS cost module, which is a Python implementation of the costing spreadsheet used to estimate the cost of proposed structural protection projects for Louisiana's 2017 Coastal Master Plan. Other colors indicate which algorithm produced a given strategy. Because we wish to minimize the total cost and expected losses, any strategy shown below and to the left of the existing system dominates the existing system, outperforming it on both metrics in the given state of the world.

The right-hand pane of Figure 1 puts the sum of system costs and expected losses on the vertical axis, allowing us to more easily identify strategies that minimize the total cost, i.e., where the expected marginal risk reduction and the marginal system cost are equalized.

Looking at strategies that dominate the existing system, we can estimate the maximum cost savings associated with a strategy that still results in the same expected losses; alternatively, we can estimate the minimum expected losses achievable by a strategy with the same cost as the existing system. These maximal improvements are summarized in Table 2.

By optimizing the system design heights, we find that under current conditions, the system cost could be reduced by $10 \%$ while maintaining the same expected annual damage. A system with the same cost could reduce expected annual damage by $73 \%$. However, this large proportional reduction is a consequence of the low baseline risk; in absolute terms, it represents an improvement of $\$ 8$ million in damage over 50 years. 
Over the three nonstationary states of the world tested, risk-minimizing strategies reduce the present value of expected damage over 50 years by an average of $31 \%$. Cost-minimizing strategies achieve equivalent risk profiles at an average of $4 \%$ lower cost ( $\$ 27$ million).

Risk-based design heights differ substantially from those of the existing system, which is designed to protect against current 100-year surge and wave events (plus some freeboard as a factor of safety). Figure 2 plots the design elevations (in NAVD88 meters) of the strategies that minimize total costs in each of the four future scenarios (noted by color), alongside the existing design elevations in black. The inset map shows the system centerlines with the existing design elevations in color, and the main panels show the length of each reach as a transect plot (noting that the eastern half of the system is longer due to the protrusions of the E North reaches). While the figure presents reach heights for the four specific optimal strategies identified by our experimental design, they are broadly representative of the designs of all strategies with lower cost and lower residual risk than the existing system: Table S1 shows the coefficient of variation in the design heights by reach (i.e., the standard deviation expressed as a proportion of the mean) for all such strategies in a given scenario. On average, the standard deviation over dominant strategies of the design elevation is only $3.4 \%$ of the optimal design elevation (when the elevation is measured relative to the topographic elevation at the foot of the levee). The figure clearly shows that if a risk-informed design paradigm has been used to develop the existing system, it would have been more efficient to have lower-than-existing crest heights on some reaches.

Surprisingly, the total cost-minimizing strategy assuming stationary risk employs design heights that are uniformly lower than the existing configuration, excepting the nearly identical height of the D North reach. Total cost-minimizing reach heights generally increase from the Low to the Medium to the High climate change scenario, though not always. This motivates a closer examination of the climate change factors driving changes in the risk-informed design heights for each reach. 
In keeping with the concept of risk-informed design, we determine what design heights for each reach, $h_{i}$ for $i \in\{1,12\}$, can efficiently achieve a policy-driven level of acceptable residual risk under various assumptions about future climate change. To this end, we solved for the Pareto frontier of efficient designs under 27 different scenarios representing every combination of the three different levels of sea level rise $s l r$, future average storm intensity int, and future storm frequency, $\alpha$, comprising the Low, Medium, and High scenarios from the previous analysis. Using the resulting design heights and performance metrics, we fit a regression model to predict the efficient height of each reach as a function of expected losses, $\mathcal{L}$, and the climate variables:

$$
h_{i}=\beta_{0 i}+\beta_{1 i} \mathcal{L}+\beta_{2 i} i n t+\beta_{3 i} \alpha+\beta_{4 i} \operatorname{slr}+\beta_{5 i} \mathcal{L} \times i n t+\beta_{6 i} \mathcal{L} \times \alpha+\beta_{7 i} \mathcal{L} \times \operatorname{slr}
$$

Table S2 details the fitted $\beta_{* i}$ coefficients for each reach and confirms that future sea level rise and storm frequency have a statistically significant impact on the efficient reach heights. The future average storm intensity generally does not. Averaging over the reaches, this model specification explains more than half of the variance in risk-based design heights $\left(\overline{r^{2}}=0.543\right)$. These results allow us to construct marginal effects describing how reach heights should change in response to new knowledge [or beliefs] about future climate change. For example, if policy makers wish to achieve a specific level of expected losses $\tilde{\mathcal{L}}$, then the marginal increase in design height corresponding to a small change in projected future sea level rise $\Delta s l r$ is, ceteris paribus, $\Delta h_{i} \approx \beta_{4 i} \Delta s l r+\beta_{7 i} \tilde{\mathcal{L}} \times \Delta s l r$.

We can visualize upgrade pathways and optimal design heights in several ways. Figure 3 shows the riskinformed design height for reach A East as a function of the desired residual risk (i.e., expected losses over the planning horizon) in the right pane, and the system cost (for all reaches) in the left pane. Each line represents a different trace of selected assumptions about future SLR and storminess. The $[S L R=$ $0.46 m, \alpha=-0.28$, int $=0.1$ ] scenario represents the best-case assumptions among the 27 scenarios tested, with the lowest sea level rise, largest reduction in the frequency of storms, and lowest increase in average storm intensity. [SLR $=0.83 m, \alpha=0$, int $=0.15]$, conversely, represents the worst-case 
scenario, while the other three scenarios depicted in Figure 3 correspond to the Low, Medium, and High scenarios from Louisiana’s 2017 Coastal Master Plan.

The optimal upgrade pathways for the A East reach in the best- and worst-case scenarios are more similar to each other than the three scenarios "in between" them with respect to the severity of assumed climate change. This is an indication of the complex spatial relationships of storm surge around the system boundary and tradeoffs between the marginal benefits of upgrades at a given cost point, wherein increasing one reach's design height requires lowering another to maintain the same cost.

Viewing the system as a whole, planners can match their subjective beliefs to either an acceptable level of risk or willingness to pay (WTP) for protection in order to identify an efficient risk-informed design. Sensitivity of the design to misspecification of future conditions can be evaluated analytically using the marginal effects, or visually by examining variation in the design heights for fixed levels of risk or WTP across different traces. For example, planners may wish to hedge against higher-than-expected SLR in a costeffective manner by adding freeboard only to reaches where optimal heights are highly sensitive to SLR (i.e., large marginal effects).

\section{Discussion}

Our best understanding of climate change impacts is that coastal hazard from storm surge-based flooding will rise ${ }^{50-53}$. Risk-informed, data-driven designs of levee and floodwall systems enable more cost-effective investments in protection. This paper provides a decision-analytic framework for levee design based on simulation of risk to the system interior. We introduce SWaMPS as a computationally efficient, physical process-based model for use in implementing our new approach. The model simulates surge/wave overtopping and system failures under varying assumptions about climate change (and/or population growth, although this uncertainty was not explored here) with sufficient accuracy to inform design decisions. The model runs quickly enough on consumer-grade computing resources to implement optimization algorithms that trace out the efficient tradeoff frontier between protection system costs and 
residual risk. It is easily modifiable to optimize alternative objectives, such as maximization of a benefitcost ratio (i.e., maximize expected loss reduction rather than minimize residual expected losses).

We show that our approach yields designs that substantially improve economic performance over systems that are based on protecting against 100-year storm surge along the system boundary. We identify many Pareto-efficient design alternatives corresponding to policy-driven levels of acceptable risk, or willingness to pay for protection, in a fraction of the time required to evaluate a single design using traditional methods and models. Our model and design framework enables planners to evaluate a much larger number of design concepts than are traditionally evaluated under a standards-based approach. Exploration of the impact of climate change factors on optimal designs indicates that optimal reach heights are sensitive to sea level rise and the frequency of tropical cyclones. The complex spatial relationships and tradeoffs between reaches demonstrate that the ability to quickly evaluate risk under a large number of design alternatives would be valuable to planners. The SWaMPS model overcomes the limitations and simplifying assumptions of other similar models while being fast enough to use with techniques for decision-making under uncertainty that require large experimental designs to identify robust designs. This will allow future work to leverage advances in decision and risk analysis in the domain of levee and floodwall system design.

\section{Methods}

This section outlines the methods and experimental design used for the analysis described in this paper. Methodological details, as well as calibration and validation information for the SWaMPS model used to execute the analysis, are provided as Supplementary Information.

The traditional standards-based approach for levee design typically considers a design-level event occurring under current conditions (i.e., the topography, bathymetry, storm frequency, and distribution of storm parameters at the time of the study). The resulting design heights may be buffered using additional freeboard, but not all studies explicitly take nonstationarity in future conditions into account. As such, we formulate risk-based design elevations in two ways: (a) assuming that current conditions persist (i.e., risk is 
stationary), and (b) considering future climate change, both over a 50-year planning horizon. (SWaMPS's "current conditions" baseline models the Louisiana coastal landscape as it existed in 2015, chosen because it was the baseline year for the state's 2017 Coastal Master Plan.) In the latter case, we examine three scenarios that vary in their assumptions about the rate of eustatic sea level rise and the average intensity and frequency of future tropical cyclones. These assumptions correspond to the Low, Medium, and High environmental scenarios from Louisiana’s 2017 Coastal Master Plan. The parameter values in Year 50 corresponding to each scenario are summarized in Table 1.

Our decision lever, $\mathbf{H}=<h_{1}, h_{2}, \ldots, h_{12}>$, defines a system configuration that sets the crest elevation of each reach $i$ to $h_{i}$ NAVD88 meters, incurring a cost $C(\mathbf{H})$ and yielding average annual losses (AAL) in year $t$ of $L(\mathbf{H}, t)(t \in \mathbb{Z}, 0 \leq t \leq 50)$. When calculating the system cost and surge/wave overtopping, we assume that floodwalls and gates are placed in the same locations and extents as the existing protection system. The twelve reaches are mapped and labeled in the inset of Figure 2.

In case (a), we minimize AAL under the current conditions landscape and calculate the expected PV of losses, with discount rate $r$, assuming AAL does not change over 50 years. In (b), we minimize the PV of the sum of AAL in each year of the planning horizon. Fischbach, et al. (2019) found that AAL in coastal Louisiana can be reasonably approximated as a piecewise linear function over time ${ }^{54}$, so for computational efficiency we explicitly model AAL every ten years in case (b) and use linear interpolation to estimate $L(\mathbf{H}, t)$ in the intervening years. In both cases, we simultaneously minimize the cost $C(\mathbf{H})$, which is also expressed as a present value and includes planning and design, construction, as well as future maintenance (future costs are also discounted at rate $r$ ). Therefore, by scalarization, our optimization problem in each case is to identify

$$
\mathbf{H}^{*}=\underset{\mathbf{H}}{\operatorname{argmin}} \omega C(\mathbf{H})+(1-\omega) \sum_{t=0}^{50} \frac{L(\mathbf{H}, t)}{(1+r)^{t}} \quad(0 \leq \omega \leq 1)
$$


We identified these risk-based design heights using the Master-Slave Borg Multi-objective Evolutionary Algorithm (MS Borg-MOEA) $)^{30,31}$, NSGA-II genetic algorithm, and a greedy algorithm that selects incremental $0.1-\mathrm{m}$ upgrades on the basis of which reach's upgrade would produce the greatest marginal cost effectiveness (i.e., marginal risk reduction divided by marginal cost). Each algorithm was used to find Pareto-efficient combinations of design elevations over the 12 levee reaches, with respect to the system cost and expected losses over a 50-year planning horizon. We repeated each optimization process with unique multiple random seeds in order to mitigate variability in the composition and quality of the nondominated solutions. The resulting Pareto frontiers from each macro replication have then been combined and sorted, discarding dominated solutions to arrive at the final frontier.

Storm surge and wave behavior for each of the 120 synthetic storms is predicted as a function of their characteristics at landfall (e.g., central pressure, radius of maximum windspeed, forward velocity, landfall location and heading). Local mean sea level varies over time in the nonstationary future scenarios and is also incorporated into the response surface. The response surface model is taken from the CLARA model ${ }^{26}$. It has been trained on a corpus of 600 storms representing 60 storms with varying landfall parameters, each run under 10 different initial sea level conditions, through a coupled ADCIRC+SWAN model using the same high-resolution mesh utilized for Louisiana's 2017 Coastal Master Plan ${ }^{55}$. Changes to the average intensity and frequency of tropical cyclones impact the statistical aggregation of individual storm results to an annual exceedance probability (AEP) curve. The former affects the probability distribution for storms' central pressure at landfall, and the latter affects the mean interarrival rate of storms used to convert the cumulative distribution function of stillwater elevations, conditional upon a storm occurring, to the AEP curve. The probabilities of system breaches are modeled as a function of surge and wave overtopping rates, with the functional relationship derived from the assumptions of a previous USACE flood risk study in coastal Louisiana ${ }^{26,56}$.

We hold other factors in the model constant throughout the planning horizon, such as population and construction standards for structure foundation heights, in order to isolate the impact of climate change 
factors on optimal design elevations. We also assume that the locations of floodwalls on top of the levee and control structures (e.g., gates, pumping stations) do not change over time.

Competing Interest Statement: The authors declare no competing interests.

\section{Data Availability}

Removed as part of double-blind peer review process. Can provide in anonymized manner upon request.

\section{Code Availability}

Removed as part of double-blind peer review process. Can provide in anonymized manner upon request.

Author Contributions: The corresponding author designed the conceptual framework and analysis plan. All authors developed the model and contributed to performing the analysis and writing the paper. 
Figures and Tables

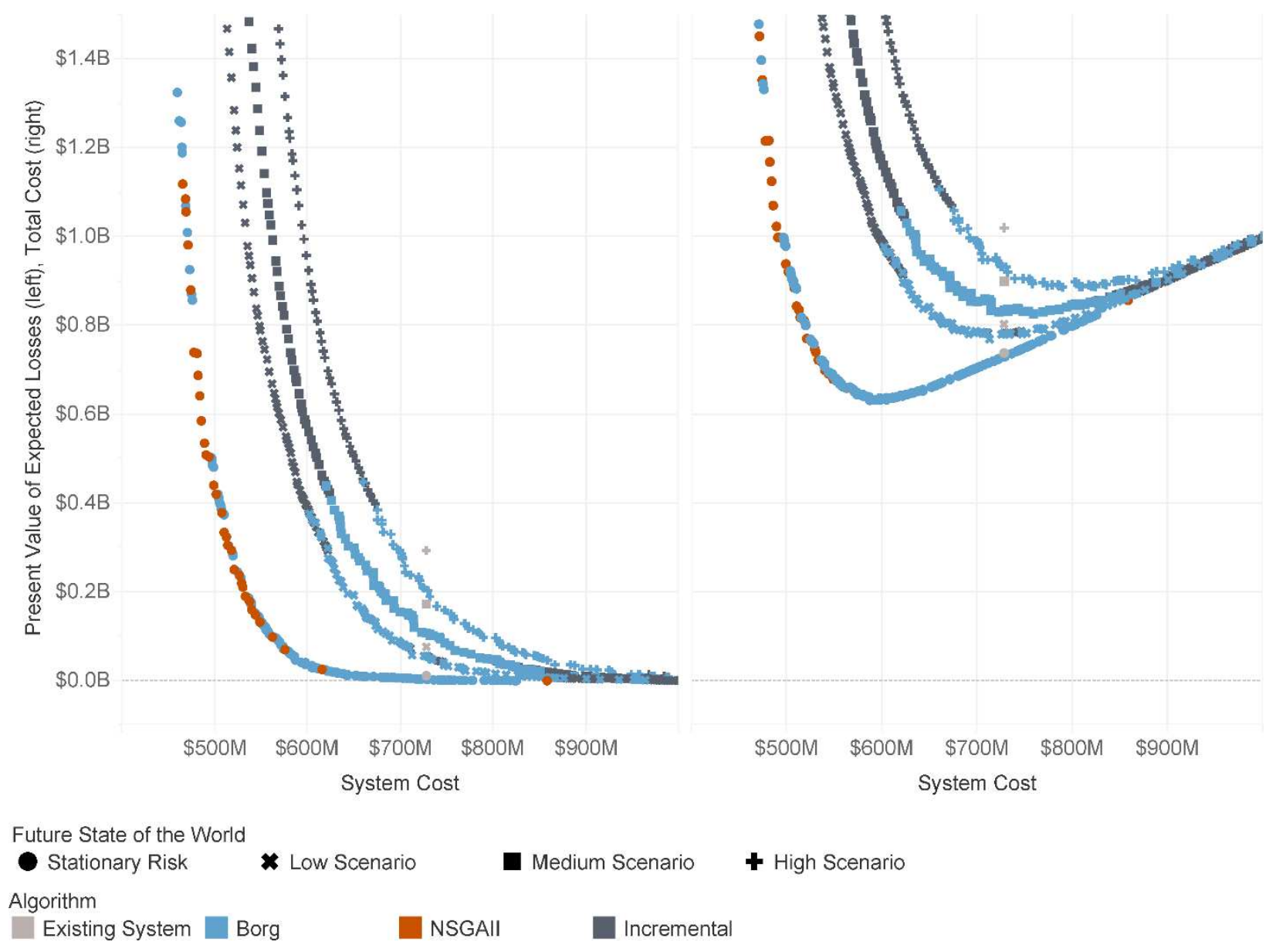

Figure 1. Pareto frontier showing performance of nondominated designs minimizing system cost and residual risk over 50 years. 


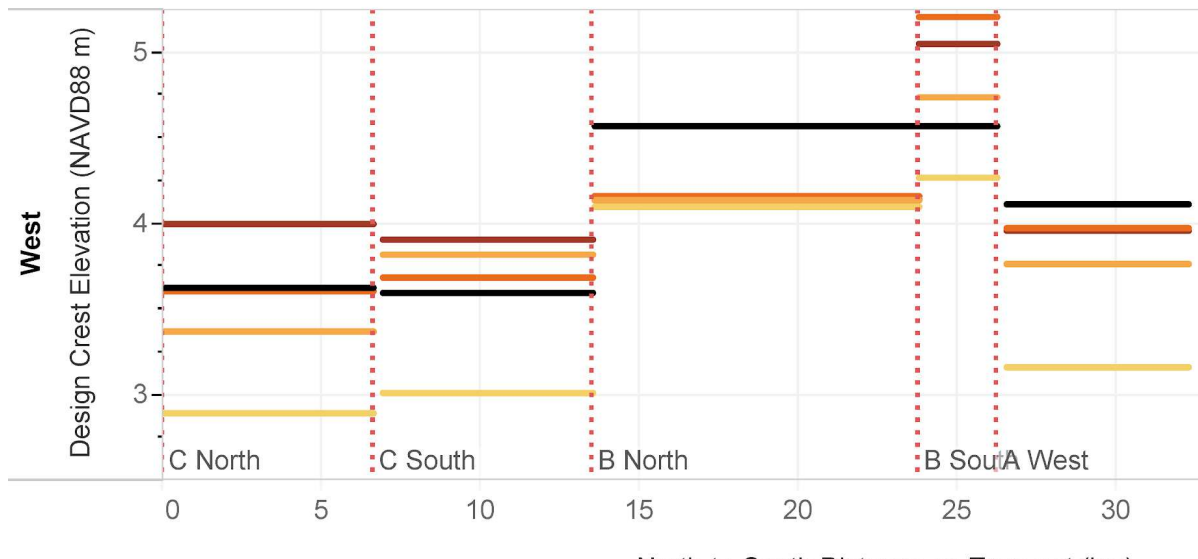

Existing Design Elevations (m)

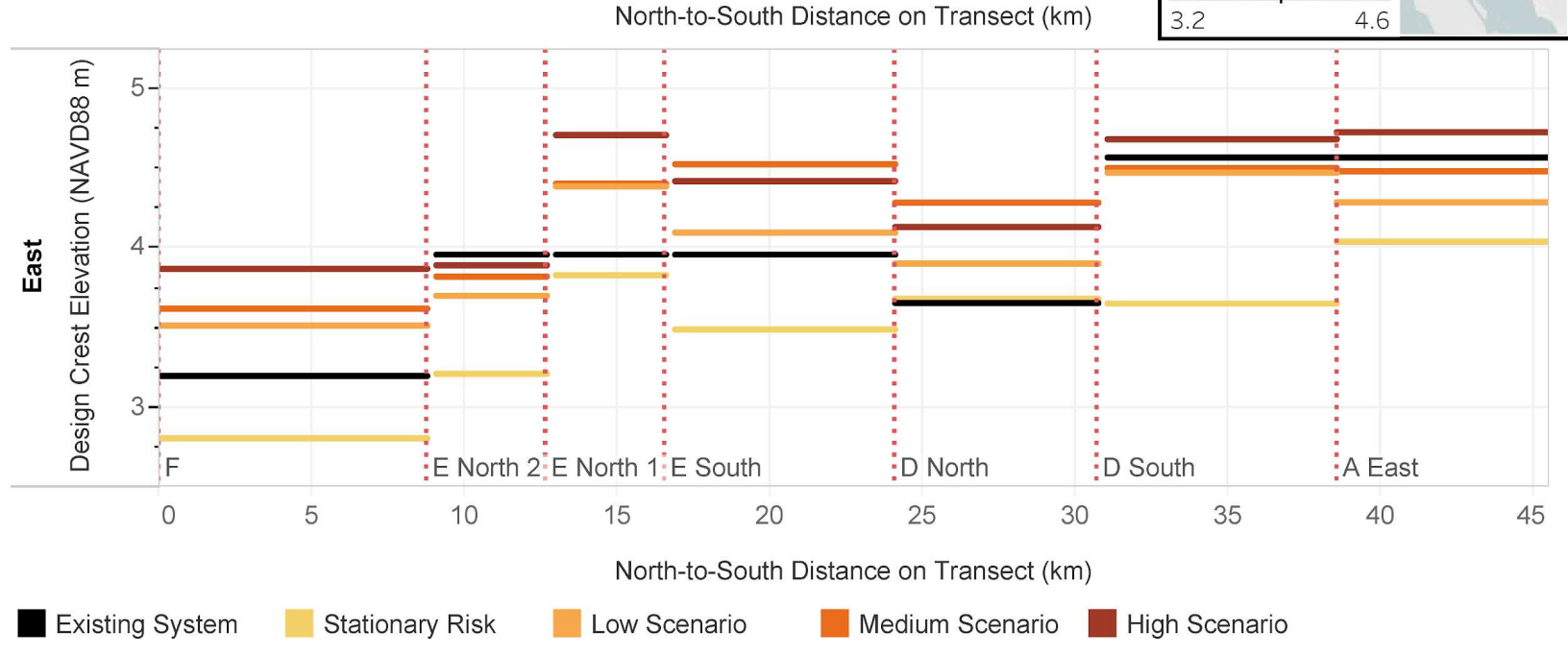

Figure 2. Optimal design heights by reach and scenario (colors), compared to existing design heights (black, also shown spatially in color by reach in the inset top-right). 

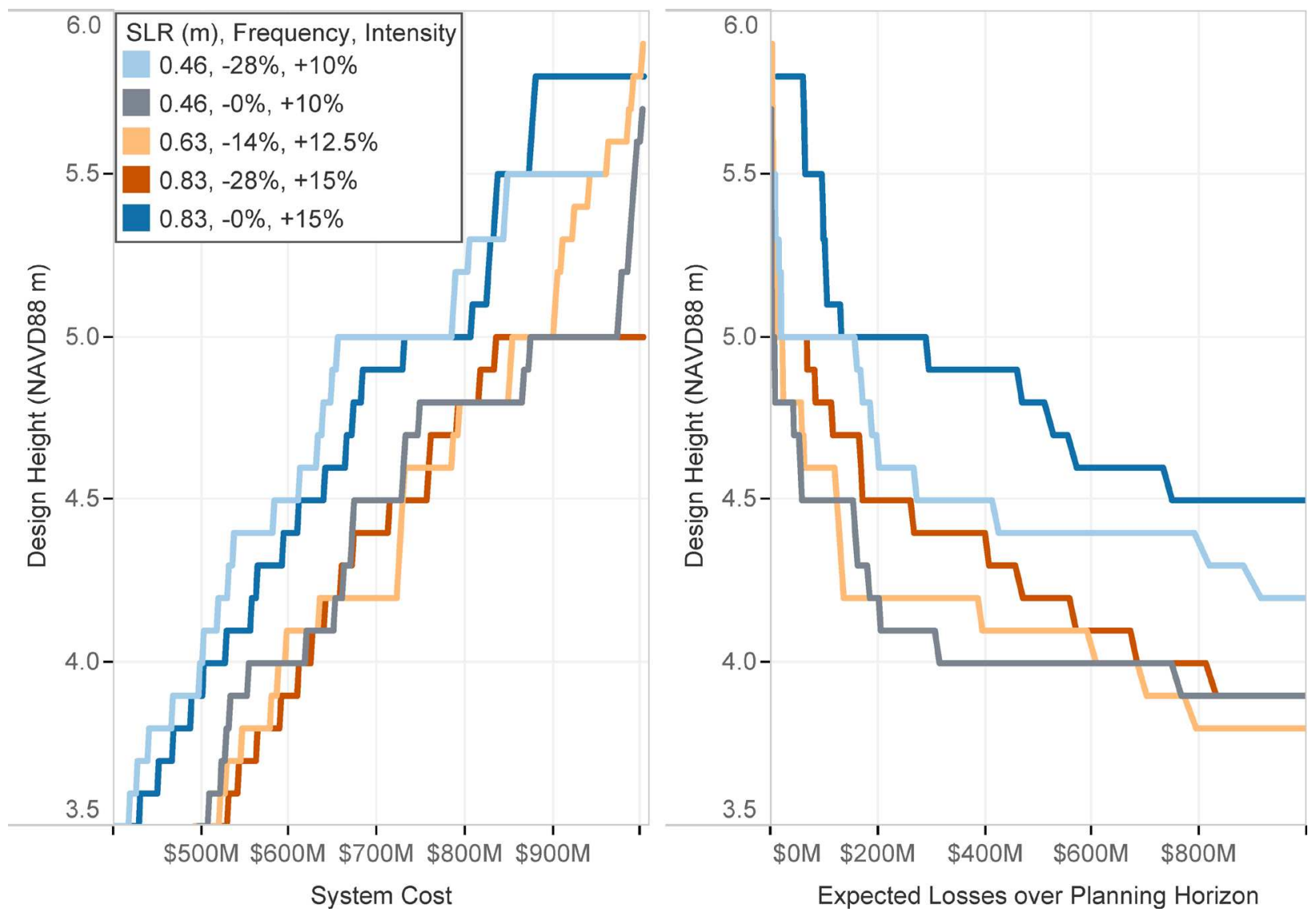

Figure 3. Risk-informed design heights for reach A East in selected climate change scenarios, as a function of the associated system cost (left) and achieved residual risk (right). 
Table 1. Climate change assumptions defining future scenarios (all values in 2065 relative to 2015).

$\begin{array}{llll} & \text { Sea Level } & \text { Storm } & \text { Storm } \\ \text { Scenario } & \text { Rise (m) } & \text { Intensity } & \text { Frequency } \\ & & & \\ \text { Low } & 0.46 & +10.0 \% & -0 \% \\ & & & \\ \text { Medium } & 0.63 & +12.5 \% & -14 \% \\ & & & \\ \text { High } & 0.83 & +15.0 \% & -28 \%\end{array}$


Table 2. Performance and improvement of cost-minimizing and risk-minimizing strategies by future scenario.

\begin{tabular}{|c|c|c|c|c|c|}
\hline & \multicolumn{5}{|c|}{ State of the World } \\
\hline & (\$ millions) & Stationary & Low & Medium & High \\
\hline Existing System & $\mathrm{PV}($ Cost $)$ & $\$ 728$ & $\$ 728$ & $\$ 728$ & $\$ 728$ \\
\hline Existing System & PV(EAD) & $\$ 11$ & $\$ 76$ & $\$ 173$ & $\$ 293$ \\
\hline Cost-Minimizing & $\mathrm{PV}(\mathrm{Cost})$ & $\$ 655$ & $\$ 709$ & $\$ 693$ & $\$ 701$ \\
\hline Risk-Minimizing & PV(EAD) & $\$ 3$ & $\$ 56$ & $\$ 108$ & $\$ 208$ \\
\hline Improvement & $\mathrm{PV}($ Cost $)$ & $10 \%$ & $3 \%$ & $5 \%$ & $4 \%$ \\
\hline Improvement & PV(EAD) & $73 \%$ & $26 \%$ & $38 \%$ & $29 \%$ \\
\hline
\end{tabular}




\section{References}

1. Montz, B. E. \& Tobin, G. A. Livin' large with levees: lessons learned and lost. Natural Hazards Review 9, 150-157 (2008).

2. Interagency Levee Policy Review Committee. The National Levee Challenge: Levees and the FEMA Flood Map Modernization Initiative. https://www.fema.gov/media-library-data/20130726-160620490-2709/levee_report_final.pdf (2006).

3. U.S. Army Corps of Engineers. Louisiana Coastal Protection and Restoration Final Technical Report: Hydraulics and Hydrology Appendix. 389 (2009).

4. U.S. Army Corps of Engineers. Risk Assessment for Flood Risk Management Studies. 25 https://www.publications.usace.army.mil/Portals/76/Users/182/86/2486/ER\%201105-2-101_Clean.pdf (2019).

5. U.S. Army Corps of Engineers. Planning Guidance Notebook. 712 https://www.publications.usace.army.mil/Portals/76/Users/182/86/2486/ER\%201105-2101_Clean.pdf?ver=ZMLDfARc21VswO1BO_VIWQ\%3d\%3d (2000).

6. Johnson, D. R. Improved methods for estimating flood depth exceedances within storm surge protection systems. Risk Analysis 39, 890-905 (2019).

7. Jacobsen, B. Hurricane surge hazard analysis: the state of the practice and recent applications for southeast Louisiana. (2013).

8. Jonkman, S. N., Kok, M., Ledden, M. V. \& Vrijling, J. K. Risk-based design of flood defence systems: a preliminary analysis of the optimal protection level for the New Orleans metropolitan area. Journal of Flood Risk Management 2, 170-181 (2009).

9. Garner, G. G. \& Keller, K. Using direct policy search to identify robust strategies in adapting to uncertain sea-level rise and storm surge. Environmental Modelling \& Software 107, 96-104 (2018).

10. Borgomeo, E. et al. Risk-based water resources planning: incorporating probabilistic nonstationary climate uncertainties. Water Resources Research 50, 6850-6873 (2014).

11. McIntyre, N. R. \& Wheater, H. S. A tool for risk-based management of surface water quality. Environmental Modelling \& Software 19, 1131-1140 (2004).

12. Wagener, T. \& Kollat, J. Numerical and visual evaluation of hydrological and environmental models using the Monte Carlo analysis toolbox. Environmental Modelling \& Software 22, 1021-1033 (2007).

13. Newman, J. P. et al. Review of literature on decision support systems for natural hazard risk reduction: Current status and future research directions. Environmental Modelling \& Software 96, 378-409 (2017).

14. Zhu, Y., Li, Y. P. \& Huang, G. H. An optimization decision support approach for risk analysis of carbon emission trading in electric power systems. Environmental modelling \& software 67, 43-56 (2015).

15. Carrasco, I. J. \& Chang, S.-Y. Random Monte Carlo simulation analysis and risk assessment for ammonia concentrations in wastewater effluent disposal. Stochastic Environmental Research and Risk Assessment 19, 134-145 (2005).

16. van Dantzig, D. Economic decision problems for flood prevention. Econometrica 24, 276-287 (1956).

17. Kind, J. M. Economically efficient flood protection standards for the Netherlands. Journal of Flood Risk Management 7, 103-117 (2014).

18. Slijkhuis, K., Van Gelder, P. \& Vrijling, J. Optimal dike height under statistical-, construction- and damage uncertainty. Structural Safety and Reliability 7, 1137-1140 (1997).

19. Eijgenraam, C. Optimal safety standards for dike-ring areas. CPB Discussion Paper https://ideas.repec.org/p/cpb/discus/62.rdf.html (2006).

20. Vrijling, J. K. Probabilistic design of water defense systems in The Netherlands. Reliability Engineering \& System Safety 74, 337-344 (2001). 
21. Sriver, R. L., Lempert, R. J., Wikman-Svahn, P. \& Keller, K. Characterizing uncertain sea-level rise projections to support investment decisions. PLOS ONE 13, e0190641 (2018).

22. Wong, T. E. \& Keller, K. Deep uncertainty surrounding coastal flood risk projections: a case study for New Orleans. Earth's Future 5, 1015-1026 (2017).

23. Wong, T. E., Bakker, A. M. R. \& Keller, K. Impacts of Antarctic fast dynamics on sea-level projections and coastal flood defense. Climatic Change 144, 347-364 (2017).

24. Ceres, R. L., Forest, C. E. \& Keller, K. Optimization of multiple storm surge risk mitigation strategies for an island City On a Wedge. Environmental Modelling \& Software 119, 341-353 (2019).

25. Hydrologic Engineering Center. HEC-FDA Flood Damage Reduction Analysis: User's Manual, Version 1.4.1. $392 \mathrm{https} / / /$ www.hec.usace.army.mil/software/hec-fda/documentation/CPD72_V1.4.1.pdf (2016).

26. Fischbach, J. R. et al. Coastal Master Plan Modeling: Attachment C3-25 - Storm Surge and Risk Assessment. (2017).

27. Resio, D. T. White paper on estimating hurricane inundation probabilities. In Performance Evaluation of the New Orleans and Southeast Louisiana Hurricane Protection System. Appendix 8: Hazard Analysis vol. VIII (U.S. Army Corps of Engineers, 2007).

28. Resio, D. T., Irish, J. \& Cialone, M. A surge response function approach to coastal hazard assessmentpart 1: basic concepts. Natural Hazards 51, 163-182 (2009).

29. Louisiana Coastal Protection and Restoration Authority. Louisiana's Comprehensive Master Plan for a Sustainable Coast. 184 https://coastal.la.gov/our-plan/2017-coastal-master-plan/ (2017).

30. Hadka, D. \& Reed, P. Borg: An auto-adaptive many-objective evolutionary computing framework. Evolutionary computation 21, 231-259 (2013).

31. Hadka, D. \& Reed, P. Large-scale parallelization of the Borg multiobjective evolutionary algorithm to enhance the management of complex environmental systems. Environmental Modelling \& Software 69, 353-369 (2015).

32. Deb, K., Pratap, A., Agarwal, S. \& Meyarivan, T. A fast and elitist multiobjective genetic algorithm: NSGA-II. IEEE transactions on evolutionary computation 6, 182-197 (2002).

33. Bioengineering ARCADIS, LLC. Larose to Golden Meadow Hurricane Protection Project: Risk Based Benefit Cost Ratio HEC-FDA Analysis. 62 (2013).

34. Meselhe, E., White, E. D. \& Reed, D. J. Coastal Master Plan Modeling: Attachment C2 - Future Scenarios. 32 (2017).

35. Emanuel, K., Sundararajan, R. \& Williams, J. Hurricanes and global warming: Results from downscaling IPCC AR4 simulations. Bulletin of the American Meteorological Society 89, 347-368 (2008).

36. Dailey, P. S., Zuba, G., Ljung, G., Dima, I. M. \& Guin, J. On the relationship between North Atlantic sea surface temperatures and US hurricane landfall risk. Journal of Applied Meteorology and Climatology 48, 111-129 (2009).

37. Vermeer, M. \& Rahmstorf, S. Global sea level linked to global temperature. Proceedings of the national academy of sciences 106, 21527-21532 (2009).

38. Murakami, H. \& Wang, B. Future change of North Atlantic tropical cyclone tracks: Projection by a 20km-mesh global atmospheric model. Journal of Climate 23, 2699-2721 (2010).

39. Church, J. A. \& White, N. J. Sea-level rise from the late 19th to the early 21 st century. Surveys in geophysics 32, 585-602 (2011).

40. Hill, K. A. \& Lackmann, G. M. The impact of future climate change on TC intensity and structure: A downscaling approach. Journal of Climate 24, 4644-4661 (2011).

41. Colbert, A. J. \& Soden, B. J. Climatological variations in North Atlantic tropical cyclone tracks. Journal of climate 25, 657-673 (2012).

42. Parris, A. S. et al. Global sea level rise scenarios for the United States National Climate Assessment. (2012).

43. Jevrejeva, S., Moore, J. C. \& Grinsted, A. Sea level projections to AD2500 with a new generation of climate change scenarios. Global and Planetary Change 80, 14-20 (2012). 
44. Church, J. A. et al. Sea level change. In Climate Change 2013: The Physical Science Basis. Contribution of Working Group I to the Fifth Assessment Report of the Intergovernmental Panel on Climate Change. ed. by: Stocker, T.F. et al. (Cambridge, UK, 1137-1216, 2013).

45. Emanuel, K. A. Downscaling CMIP5 climate models shows increased tropical cyclone activity over the 21st century. Proceedings of the National Academy of Sciences 110, 12219-12224 (2013).

46. Colbert, A. J., Soden, B. J., Vecchi, G. A. \& Kirtman, B. P. The impact of anthropogenic climate change on North Atlantic tropical cyclone tracks. Journal of climate 26, 4088-4095 (2013).

47. Kossin, J. P., Olander, T. L. \& Knapp, K. R. Trend analysis with a new global record of tropical cyclone intensity. Journal of Climate 26, 9960-9976 (2013).

48. Villarini, G. \& Vecchi, G. A. Twenty-first-century projections of North Atlantic tropical storms from CMIP5 models. Nature Climate Change 2, 604-607 (2012).

49. U.S. Army Corps of Engineers. Economic Guidance Memorandum, 20-01, Federal Interest Rates for Corps of Engineers Projects for Fiscal Year 2020. 13 (2020).

50. Nicholls, R. J. \& Small, C. Improved estimates of coastal population and exposure to hazards released. Eos, Transactions American Geophysical Union 83, 301-305 (2002).

51. Condon, A. J. \& Sheng, Y. P. Evaluation of coastal inundation hazard for present and future climates. Natural hazards 62, 345-373 (2012).

52. Hallegatte, S., Green, C., Nicholls, R. J. \& Corfee-Morlot, J. Future flood losses in major coastal cities. Nature Climate Change 3, 802-806 (2013).

53. Vousdoukas, M. I. et al. Climatic and socioeconomic controls of future coastal flood risk in Europe. Nature Climate Change 8, 776-780 (2018).

54. Fischbach, J. R., Johnson, D. R. \& Groves, D. G. Flood damage reduction benefits and costs in Louisiana's 2017 Coastal Master Plan. Environmental Research Communications 1, 111001 (2019).

55. Roberts, H. J. \& Cobell, Z. Coastal Master Plan Modeling: Attachment C3-25.1 - Storm Surge. 110 (2017).

56. Interagency Performance Evaluation Taskforce. Performance Evaluation of the New Orleans and Southeast Louisiana Hurricane Protection System, Vol. VIII: Engineering and Operational Risk and Reliability Analysis. (2009). 
Figures

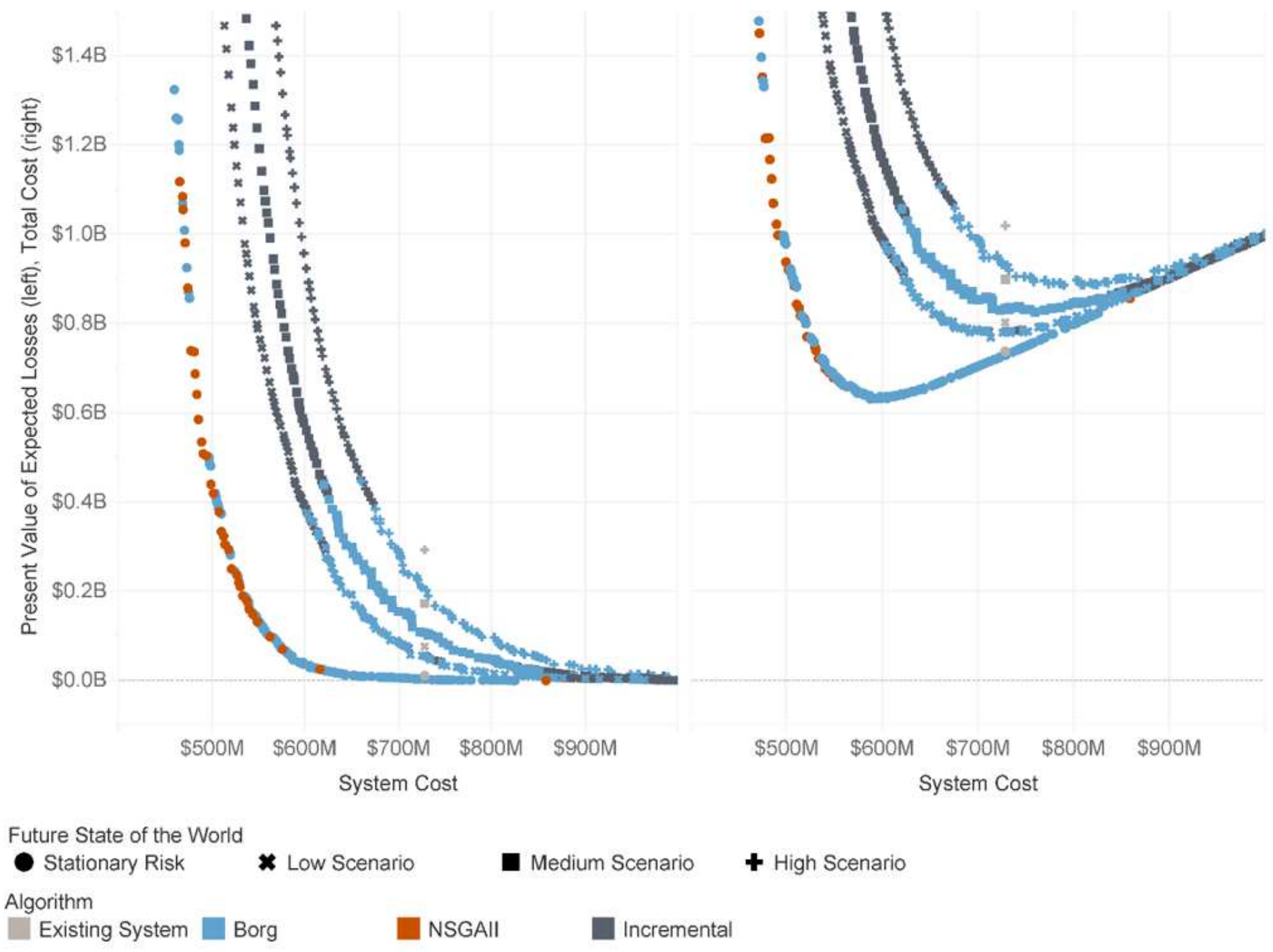

\section{Figure 1}

Pareto frontier showing performance of nondominated designs minimizing system cost and residual risk over 50 years. 
Side of Bayou Lafourche

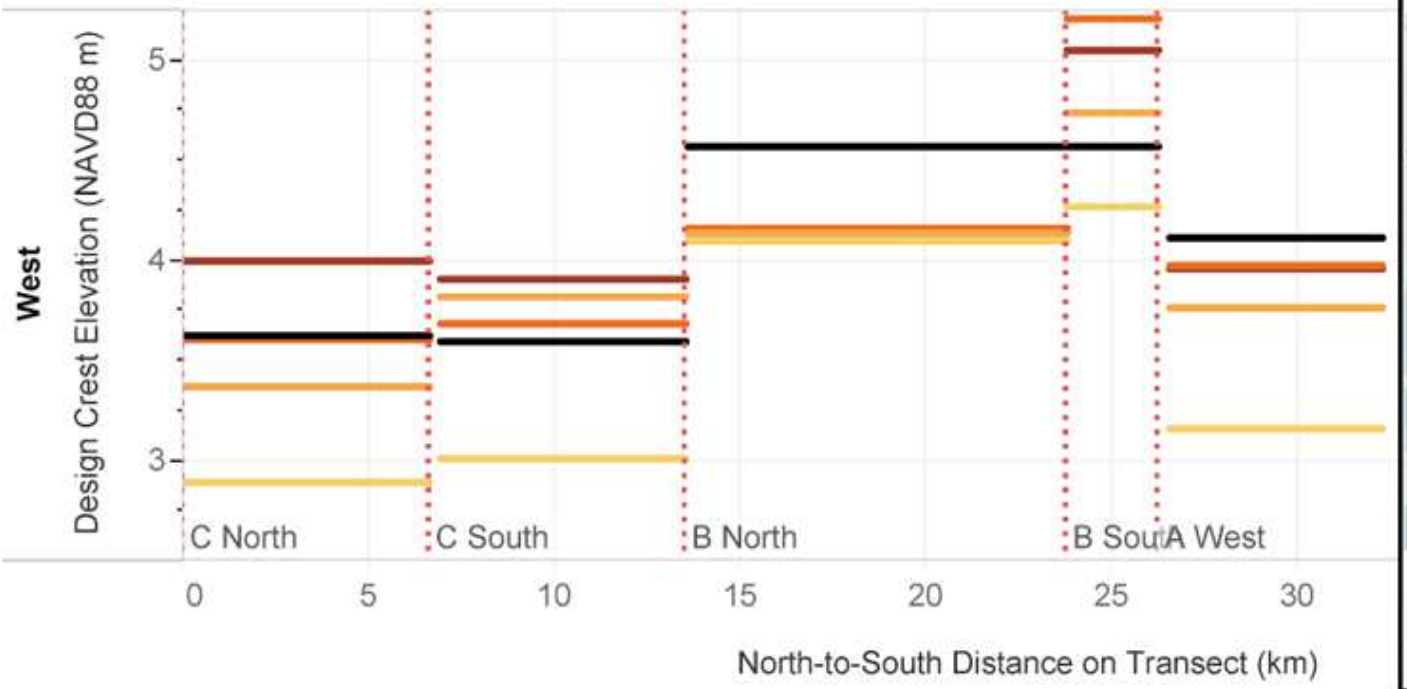

Existing Design Elevations ( $m$ )

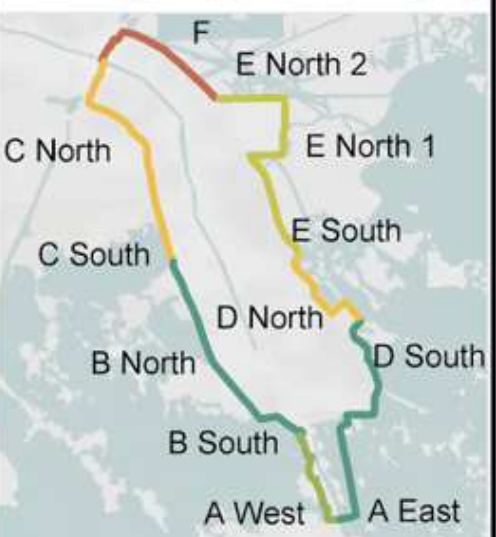

Crest Elevation $(\mathrm{m})$

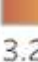

4.6

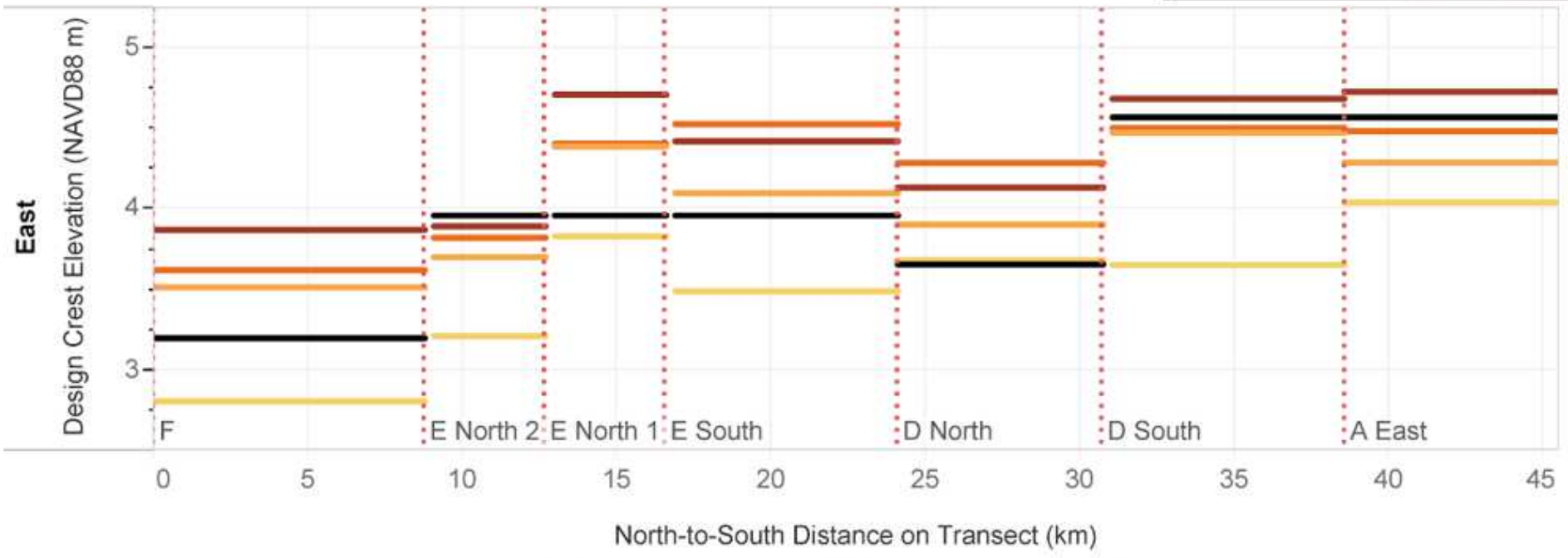

Existing System

Stationary Risk

Low Scenario

Medium Scenario

High Scenario

Figure 2

Optimal design heights by reach and scenario (colors), compared to existing design heights (black, also shown spatially in color by reach in the inset top-right). 

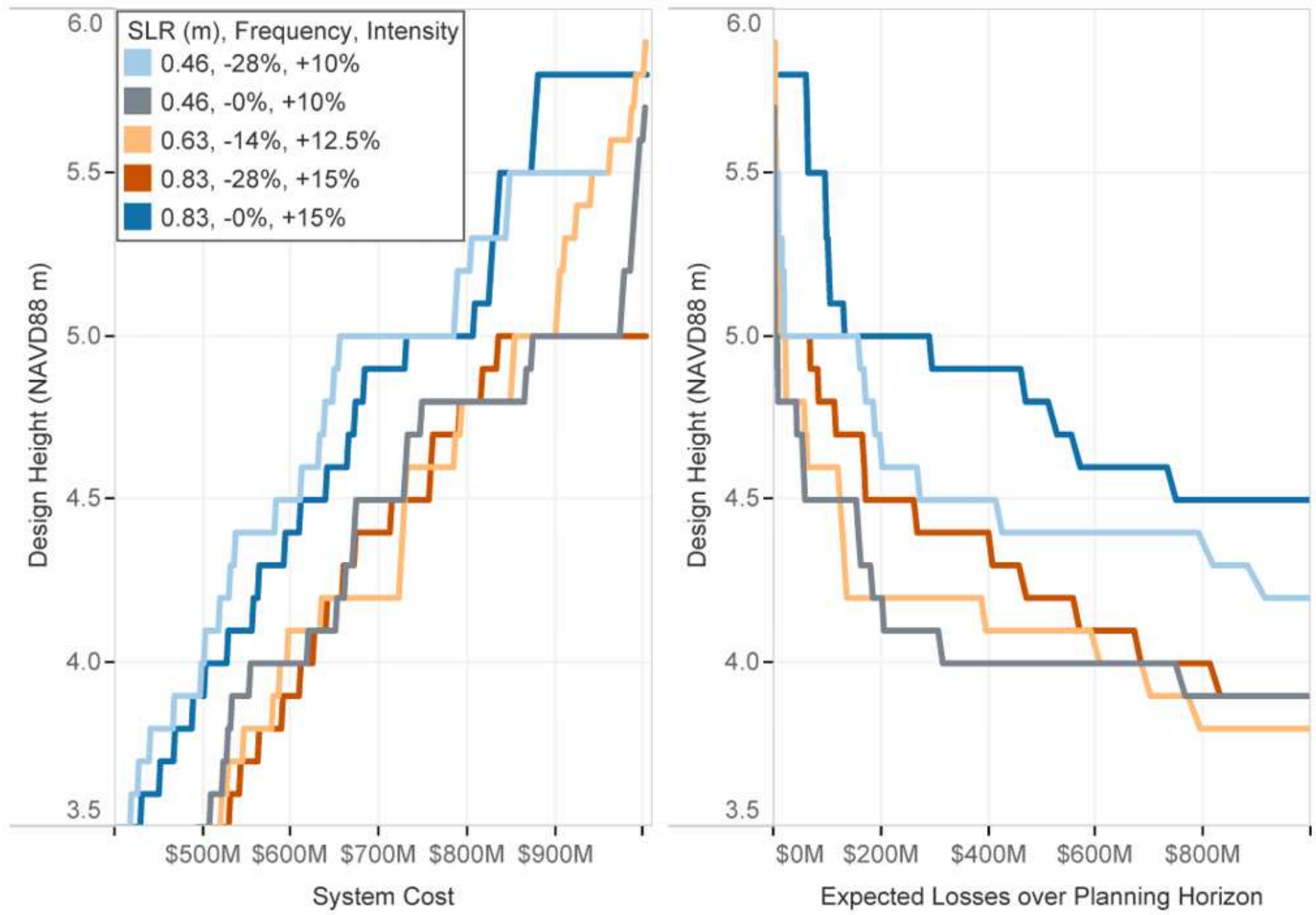

Figure 3

Risk-informed design heights for reach A East in selected climate change scenarios, as a function of the associated system cost (left) and achieved residual risk (right).

\section{Supplementary Files}

This is a list of supplementary files associated with this preprint. Click to download.

- SWaMPSSI20210217.pdf 